\title{
Self-dependent Equivalent Circuit Modeling of Electrostatic Comb Transducers for Integrated MEMS
}

\author{
Toshiyuki Tsuchiya, Hiroyuki Tokusaki, Yoshikazu Hirai, Koji Sugano, \\ and Osamu Tabata
}

Kyoto University, Department of Micro Engineering, Yoshida-Honmachi, Sakyo-ku, 606-8501 Kyoto, Japan

\{tutti,hirai, sugano, tabata\}@me.kyoto-u.ac.jp

\begin{abstract}
The article presents a new equivalent circuit model of electrostatic comb transducers for an integration of MEMS into LSI. The model detects the displacement using a dummy spring to express the current induced by the movement, and the model parameters can be derived from only its dimensions not from external parameters, such as acting force and applied voltage. In addition, being composed from basic circuit components, the model is easily utilized in conventional circuit simulation software. The in-plane two-degreeof-freedom comb model was applied to a simple SOI coupled resonator and the measured and simulated electrical and mechanical properties showed good agreement with each other. The model was also applied to a decoupled two-axis resonator for a vibrating gyroscope made on silicon-on-glass substrate. Frequency tuning and oscillating circuit with constant amplitude control have been tested and the tuning results and transient responses showed good agreement as well.
\end{abstract}

Keywords: electrostatic comb transducers, equivalent circuit, resonator, oscillation, circuit simulation.

\section{Introduction}

In recent years, the downscaling of semiconductor devices is going to approach to the quantum limit and new technologies have been proposed for further performance improvements to extend the Moore's law to the future. Micro-electro-mechanical system (MEMS) is one of the solutions for breaking the limit by substituting circuit blocks with active and passive MEMS components, such as variable capacitors, RF filters, and switches. These components consist of mechanical, electrical, and electromechanical parts integrated with circuits.

On designing not only semiconductor devices but also MEMS, simulations are crucial for highly integrated devices and coupled analysis of electrical and mechanical domains is strongly demanded for ease of MEMS designing. Electrical equivalent circuit analysis is a good solution for this demand, since we can easily utilize existing tools for designing electronics. Mechanical structures are expressed with lumped parameter systems, such that a mass, spring, and dumper are simply converted to an 
inductor, capacitor, and resistor, respectively. Electro-mechanical transducers, such as piezoelectric, electrostatic, thermal, and electromagnetic have been expressed with simple transformers or controlled voltage/current sources. This substitution is valid for small signal ac analysis with a fixed dc operating point. Since the parameters of transformer are variable depending on their operating conditions, such as dc bias, force, and displacement, we need to change the circuit parameter whenever the modifying other components and it is difficult to perform transient analysis.

In this research, we focused on an electrostatic comb transducer. The electrostatic MEMS has been widely used for mechanical sensors and optical actuators and its compatibility to CMOS LSI on fabrication and operation is good. The comb (interdigitated cantilever electrodes) transducer has been widely utilized to these devices. Various models of comb transducer have been reported. Nguyen et al. reported an equivalent circuit of three port comb resonator in which the mechanical parts and transducers are described. In this model, the circuit parameters are varied with de biases [1]. A method for composing equivalent circuit of arbitral comb transducers up to three degree of freedom has been reported to efficiently reconstruct component models using Lagrange equation [2][3]. However, they also formulate the admittance after the system is decided. In these methods, the transducer characteristic is linearized at the equilibrium position balanced by connected springs to the transducer and applied force like electrostatic or external forces. Rebuilding of the equivalent circuit is required whenever the connected mechanical structure and electrical circuit are changed. To solve this problem, a new component modeling of the electromechanical transducer independent from connected system parameters is required. We proposed a new comb transducer model only depending on the dimensions of comb electrodes and physical constants.

In this article, the concept of the self-dependent model is described first, and the equivalent circuit analysis and experimental validation of two-degree-of freedom (2DOF) coupled resonator are shown. Then the frequency tuning analysis and the transient analysis of oscillating circuit for a $2 \mathrm{DOF}$ decoupled resonator as a vibrating gyroscope is described.

\section{$2 \quad$ Self-dependent Comb Transducer Model [4]}

\subsection{Equivalent Circuit Modeling of Electrostatic Transducer}

Figure 1a shows a one-degree-of-freedom (1DOF) comb transducer model, having a uniform gap between opposing comb fingers of $d$, structure thickness of $b$, initial overlap of comb fingers of $x_{0}$, and the number of comb fingers of $n$. A mechanical system of impedance $Z_{\mathrm{m}}$ is connected to the movable electrode. $x$ is a displacement of the comb transducer. The capacitance $C(x)$ of comb fingers is given by

$$
C(x)=\frac{2 n \varepsilon b\left(x_{0}+x\right)}{d},
$$

where $\varepsilon$ is the dielectric constant. The electrostatic force $F$ along $x$-direction is equal to the negative derivative with respect to $x$ of the electrostatic energy stored the capacitance, 


$$
F=\frac{1}{2} \frac{\partial C(x)}{\partial x} E^{2}=\frac{n \varepsilon b}{d} E^{2},
$$

where $E$ is the applied voltage between opposing comb fingers. The electrical charge $Q$ is given by

$$
Q=C(x) E=\frac{2 n \varepsilon b\left(x_{0}+x\right)}{d} E,
$$

The current $i$ on the comb transducer is temporal differentiation of the electrical charge $Q$ as follows

$$
i=\frac{\partial Q}{\partial t}=\frac{2 n \varepsilon b E}{d} \frac{d x}{d t}+C(x) \frac{d E}{d t} .
$$

In conventional equivalent circuit model, as shown in Fig. 1b, these electro-mechanical transductions are described as a simple transformer of electro-mechanical coupling coefficient $A$;

$$
A=\frac{\partial F}{\partial E}=\frac{2 n \varepsilon b E}{d} .
$$

This is simple and useful for small signal ac analysis on fixed dc bias condition. The mechanical responses are probed by the current on the mechanical impedance $Z_{\mathrm{m}}$.

a)
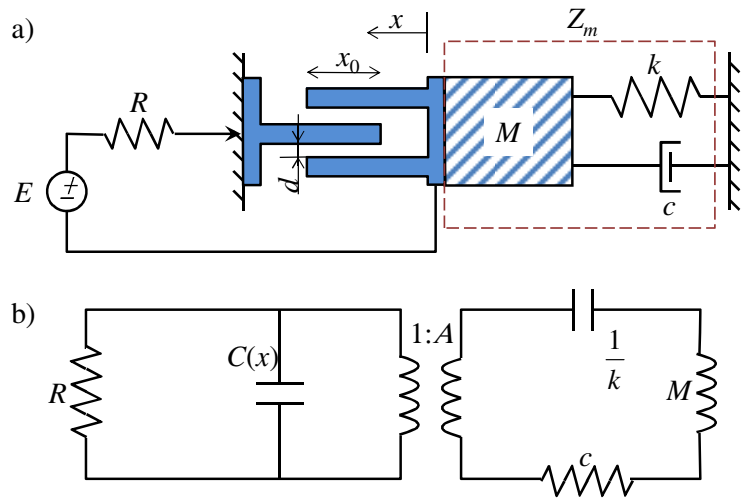

Fig. 1. Simple equivalent circuit model of one-degree-of-freedom comb resonator

\subsection{Self-dependent Equivalent Circuit Modeling of 1DOF Comb Transducer}

The parameters in the model that is shown in Fig. 1 shall be changed when the equilibrium position of the comb fingers is changed. That is happen on changing the components in the circuit and inputs, such as dc bias voltage and applied force, since the coupling coefficient $A$ and the capacitance $C(x)$ depends on $E$ and $x$, respectively. We need to recalculate the comb displacement $x$ and put the parameters on the circuit, which is not convenient and makes the transient analysis difficult. 
To develop a new self-dependent model, the current $i$ on the comb transducer is described in a different expression as follows,

$$
\begin{gathered}
i=\frac{\partial Q}{\partial t}=i_{0}+i_{11}+i_{12}, \\
i_{0}=C_{0} \frac{d E}{d t}, \\
i_{11}=\frac{2 n \varepsilon b}{d} v E, \\
i_{12}=\frac{2 n \varepsilon b x}{d} \frac{d E}{d t}=\frac{i_{0}}{x_{0}} x,
\end{gathered}
$$

where $C_{0}$ is equal to $C(0)$ which shows initial capacitance of comb transducer and $v$ is the velocity of comb transducer. The current is separated into three terms. $i_{0}$ is the current on the initial capacitance $C_{0} . i_{11}$ is the current generated by the motion of the comb electrodes, which is proportional to the velocity $v . i_{12}$ is the current on the changed capacitance, which is proportional to the displacement $x$ and $i_{0}$.

The model was designed by describing the equations 2 and 6 . To express electrostatic force generated by applied voltage on the comb, a voltage source that outputs a force (voltage) proportional to square of $E$ was adopted. For the current on the comb, the first two terms $i_{0}$ and $i_{11}$ in Eq. 6 can be described with a capacitance and a current source, respectively. The current from the source is proportional to the product of $v$ and $E$, which are directly probed from the circuit. However, the third term $i_{12}$, which represents a current caused by capacitance change proportional to the displacement $x$, is hard to be described, since there is no component showing the displacement of the comb. In the new model, we inserted a dummy spring $k^{*}$ into the mechanical circuit, which means that the spring connected between combs as shown in Fig. 2a. The spring constant $k^{*}$ is small enough to be neglected on response but it detects a force (voltage) on the dummy spring and the displacement $x$ is obtained from the force. In summary, Fig. 3 shows the composed equivalent circuit.

a)

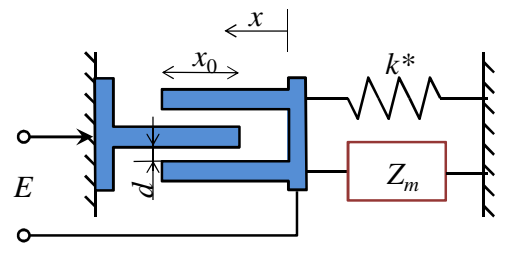

b)

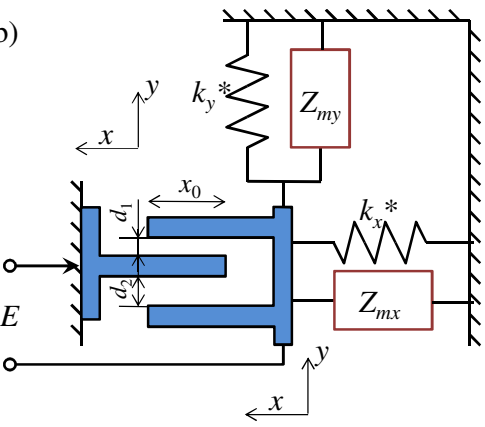

Fig. 2. a) 1 DOF and b) $2 \mathrm{DOF}$ comb transducer with dummy springs 


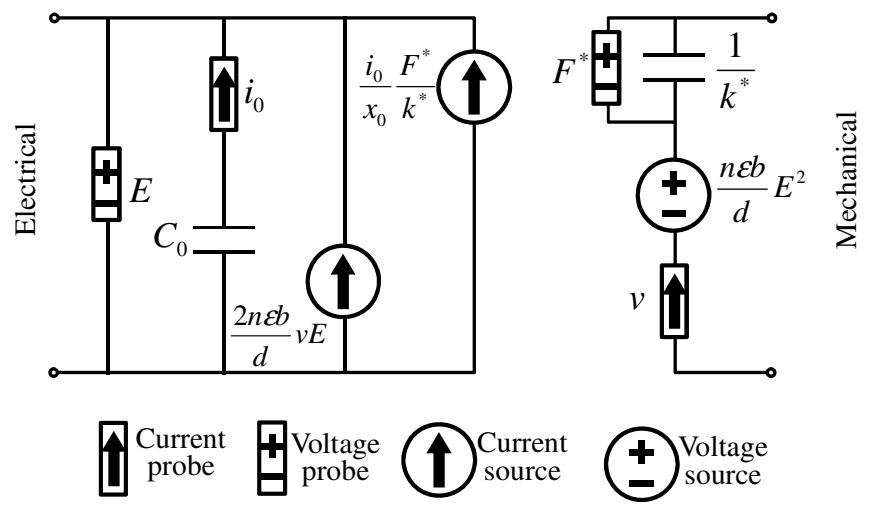

Fig. 3. Self-dependent equivalent circuit model of the 1DOF comb transducer

\subsection{Two-Degree-of-Freedom in-Plane Comb Model}

The self-dependent model was extended to an in-plane two-degree-of-freedom (2DOF) comb transducer, shown in Fig. 1b. The comb structure moves in-plane two directions and has asymmetrical gaps to detect and activate along gap $(y)$ direction. The capacitance $C(x, y)$ is

$$
C(x, y)=\frac{n \varepsilon b\left(x_{0}+x\right)}{d_{1}-y}+\frac{n \varepsilon b\left(x_{0}+x\right)}{d_{2}+y},
$$

where $d_{1}$ and $d_{2}$ are the gaps of asymmetric combs $\left(d_{1}<d_{2}\right)$. In this case, the displacement $y$ is in denominator. $C(x, y)$ was described with the Taylor series to avoid the dividing operation by the displacement $y$ in the circuit, assuming $y$ is small compared to the initial gaps. Then, the electrostatic forces along $x$ (along finger) and $y$ (gap) directions are described as;

$$
\begin{gathered}
F_{x}=X_{1} E^{2}+X_{2} y E^{2} \\
\left\{X_{1}=\frac{1}{2}\left(\frac{n \varepsilon b}{d_{1}}+\frac{n \varepsilon b}{d_{2}}\right), X_{2}=\frac{1}{2}\left(\frac{n \varepsilon b}{d_{1}{ }^{2}}-\frac{n \varepsilon b}{d_{2}{ }^{2}}\right)\right. \\
F_{y}=Y_{1} E^{2}+Y_{2} x E^{2}+Y_{3} y E^{2}, \\
\left\{\begin{array}{l}
Y_{1}=\frac{1}{2}\left(\frac{n \varepsilon b x_{0}}{d_{1}{ }^{2}}-\frac{n \varepsilon b x_{0}}{d_{2}{ }^{2}}\right), Y_{2}=\frac{1}{2}\left(\frac{n \varepsilon b}{d_{1}{ }^{2}}-\frac{n \varepsilon b}{d_{2}{ }^{2}}\right) x \\
Y_{3}=\frac{n \varepsilon b x_{0}}{d_{1}{ }^{3}}+\frac{n \varepsilon b x_{0}}{d_{2}{ }^{3}}
\end{array}\right.
\end{gathered}
$$

The current on the electrode is described as; 


$$
\begin{aligned}
& i=\left(I_{11} x+I_{12} y\right) i_{0}+\left(I_{21}+I_{22} y\right) v_{x} E+\left(I_{31}+I_{32} x+I_{33} y\right) v_{y} E, \\
& I_{11}=\left(\frac{n \varepsilon b}{d_{1}}+\frac{n \varepsilon b}{d_{2}}\right) \frac{1}{C_{0}}, I_{12}=\left(\frac{n \varepsilon b x_{0}}{d_{1}^{2}}-\frac{n \varepsilon b x_{0}}{d_{2}^{2}}\right) \frac{1}{C_{0}} \\
& I_{21}=\frac{n \varepsilon b}{d_{1}}+\frac{n \varepsilon b}{d_{2}}, I_{22}=\frac{n \varepsilon b}{d_{1}{ }^{2}}-\frac{n \varepsilon b}{d_{2}{ }^{2}} \\
& I_{31}=\frac{n \varepsilon b x_{0}}{d_{1}{ }^{2}}-\frac{n \varepsilon b x_{0}}{d_{2}{ }^{2}}, I_{32}=\frac{n \varepsilon b}{d_{1}{ }^{2}}-\frac{n \varepsilon b}{d_{2}{ }^{2}} \\
& I_{33}=2\left(\frac{n \varepsilon b x_{0}}{d_{1}{ }^{3}}+\frac{n \varepsilon b x_{0}}{d_{2}{ }^{3}}\right)
\end{aligned}
$$

Using eqs. 11, 12 and 13, a component model for 2DOF comb transducer was composed. The displacements $x$ and $y$ were detected using two dummy springs, as shown in Fig. 2b. The model is shown in Fig. 4.

The proposed equivalent circuit models can be easily implemented to circuit simulation software, since the models are described only with components provided in these software packages. In addition, the parameters of the components are determined only with the dimensions of the combs. When the comb structure has been decided, the model can be used without the modification of the parameter, even if other components both in electrical and mechanical circuits are changed.

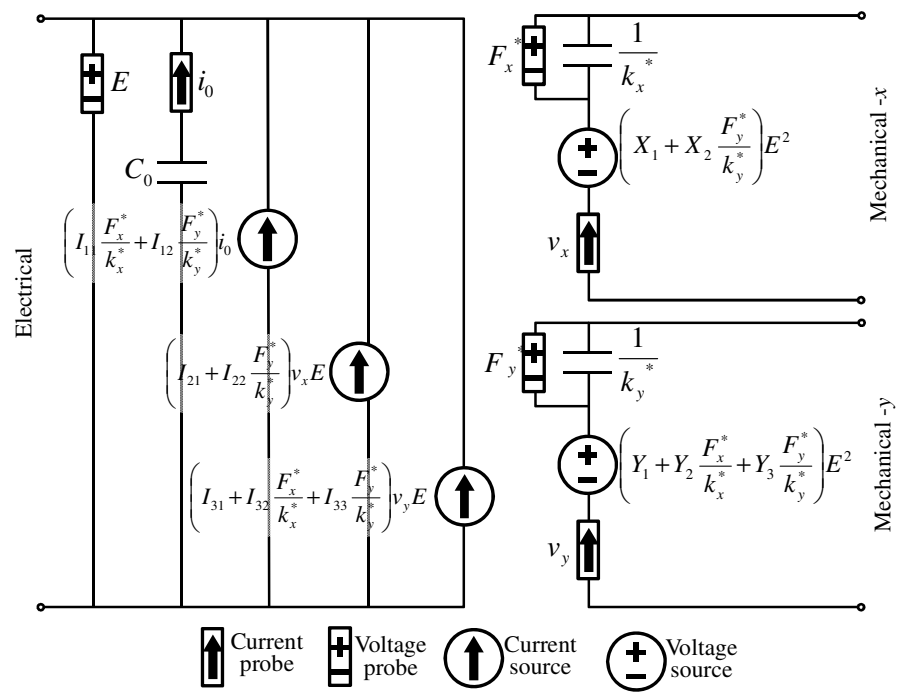

Fig. 4. Equivalent circuit model of the 2DOF comb transducer

\section{Two-Degree-of-Freedom Coupled Resonator}

\subsection{Experiments}

The 2DOF model was applied to a simple in-plane resonator fabricated on an SOI wafer. An asymmetric comb transducer is attached on the resonator mass supported 
with four meander springs. The structure is $20 \mu \mathrm{m}$ thick and the springs are $5 \mu \mathrm{m}$ wide. It moves along in-plane $x$ and $y$ directions. Fig. 5 shows the fabricated device and its equivalent circuit. The mechanical part of the equivalent circuit has two loops which corresponds $x$ and $y$ motions. The mass (inductor) parameters are common but those of the springs (capacitances) and dampers (resisters) have different values. In the electrical part, an additional capacitance was connected in parallel to comb input, which shows the parasitic capacitance through the substrate.

The measurement and simulation were performed by applying a small ac voltage (1 V) with dc bias $(5,6$, and $7 \mathrm{~V})$, in vacuum $(\sim 20 \mathrm{~Pa})$. The electrical responses were measured using an impedance analyzer (Agilent, 4294A). The mechanical responses were measured using a stroboscopic microscope (Polytec, MSA400). The model parameters of comb transducers were derived from the dimensions in the design as shown in Table I. The ac responses of equivalent circuit were simulated using a spice software (National Instruments, MultiSim). The lumped parameters of mechanical components were fitted to the measured responses at $5 \mathrm{~V}$ of dc bias, while the mass was fixed to the designed value.
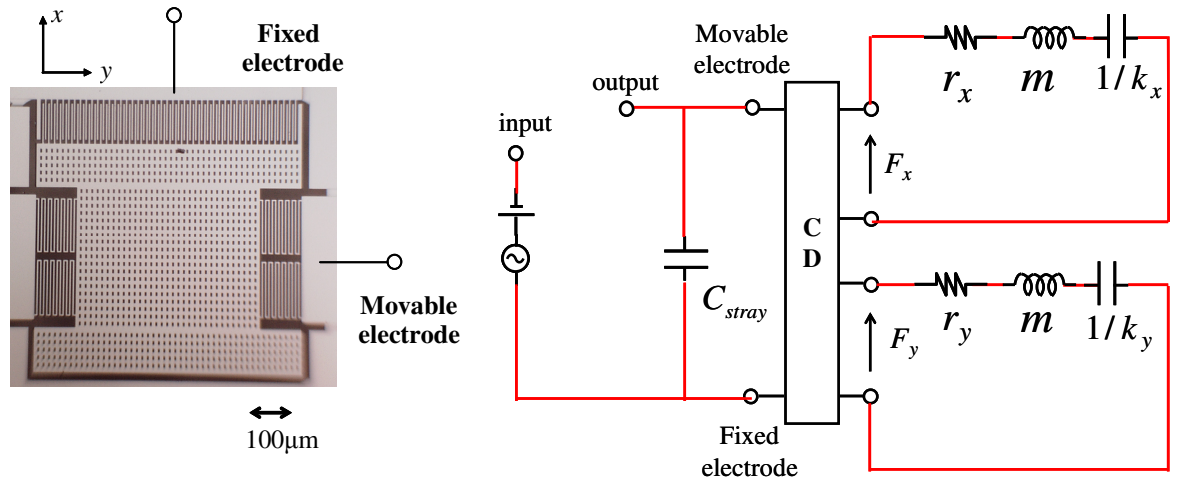

Fig. 5. In-plane 2DOF SOI resonator

Table 1. Parameters of the equivalent circuit

\begin{tabular}{lcc}
\hline Initial gap & $d_{1}$ & $3 \mu \mathrm{m}$ \\
Initial gap & $d_{2}$ & $5 \mu \mathrm{m}$ \\
Initial overlap & $x_{0}$ & $100 \mu \mathrm{m}$ \\
Structure height & $b$ & $20 \mu \mathrm{m}$ \\
The number of combs & $n$ & 45 \\
Mass & $m$ & $1.54 \times 10^{-8} \mathrm{~kg}$ \\
Spring constant ( $x$-axis) & $k_{x}$ & $25.05 \mathrm{~N} / \mathrm{m}$ \\
Spring constant (y-axis) & $k_{y}$ & $13.17 \mathrm{~N} / \mathrm{m}$ \\
Damping (x-axis) & $r_{x}$ & $2.3 \times 10^{-6} \mathrm{Ns} / \mathrm{m}$ \\
Damping (y-axis) & $r_{y}$ & $37 \times 10^{-6} \mathrm{Ns} / \mathrm{m}$ \\
Stray capacitance & $C_{\text {stray }}$ & $2.45 \mathrm{pF}$ \\
\hline
\end{tabular}

\subsection{Frequency Responses}

The measured resonant frequencies of $x$ (along comb fingers) and $y$ (gap direction) axis were 6.4 and $4.4 \mathrm{kHz}$, respectively. An in-plane rotation $\left(\theta_{\mathrm{z}}\right)$ mode was observed at 
7.2 kHz. Generally, both the mechanical and electrical responses are matched well between measurement and simulation at all the dc bias voltages, which indicates the proposed model describes the frequency responses properly. However, on the electrical measurement, the resonant peaks are not clear, especially for the $x$-mode, which is caused by a relatively large parasitic capacitance which dominates the impedance properties.

(a)

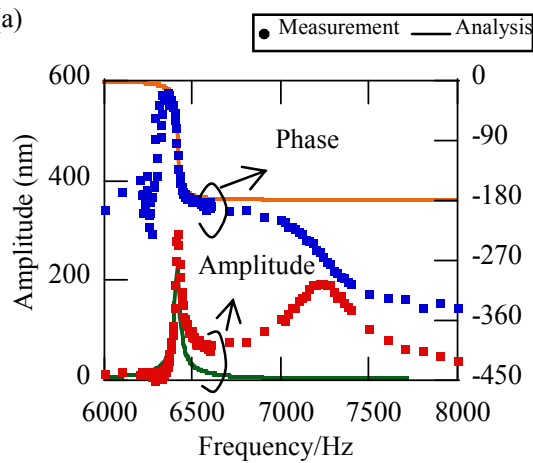

(c)

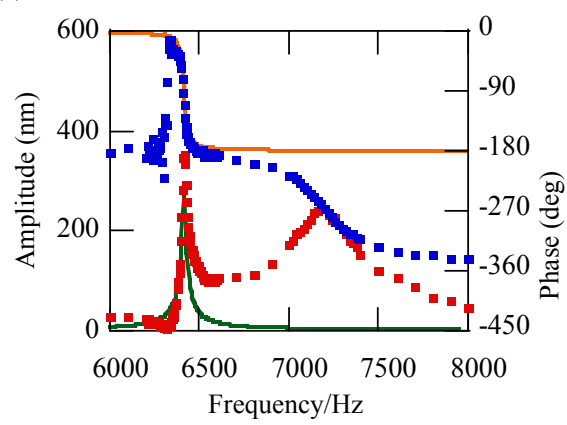

(e)

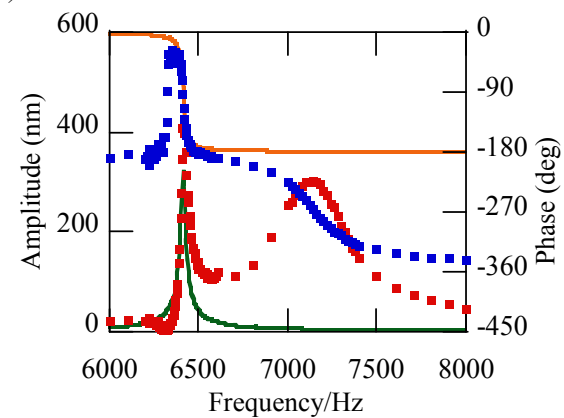

(b)

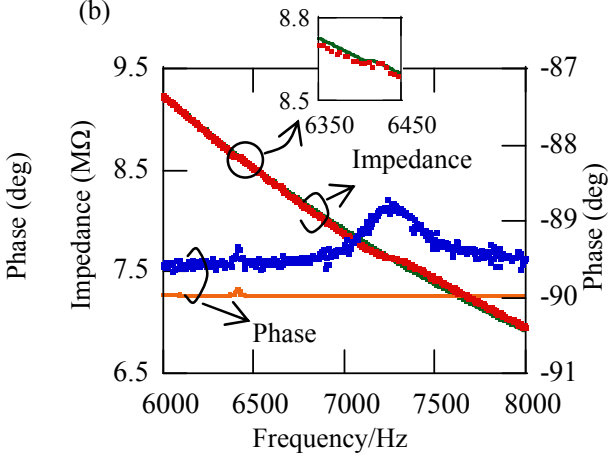

(d)

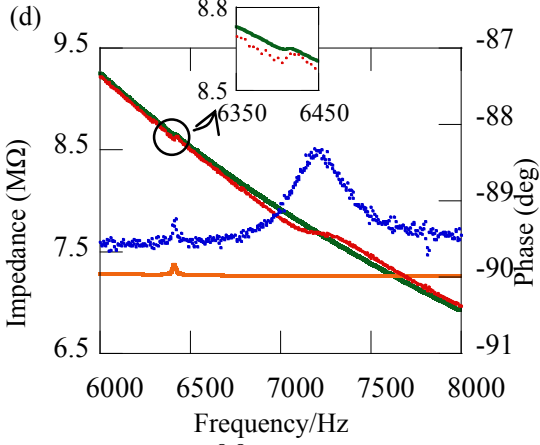

(f)

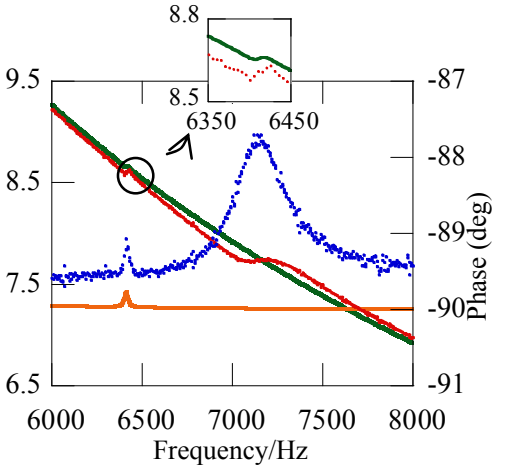

Fig. 6. Mechanical (a, c, e) and electrical (b, d, f) frequency responses around $x$-axis resonance mode at bias voltage $(\mathrm{a}, \mathrm{b}) 5 \mathrm{~V},(\mathrm{c}, \mathrm{d}) 6 \mathrm{~V},(\mathrm{e}, \mathrm{f}) 7 \mathrm{~V}$

The mechanical and electrical frequency responses at the different dc biases near the $x$ and $\theta_{\mathrm{z}}$ modes were plotted in Figs. 6 . The mechanical responses in the region near $x$ mode of $6.4 \mathrm{kHz}$ showed good agreement between the simulation and measurement, 
whereas those of the other region in Fig. 6a, 6c, 6e are dominated mainly by the inplane rotation mode $\left(\theta_{\mathrm{z}}\right)$. The $x$-axis resonant frequency does not depend on the $\mathrm{dc}$ bias, since the electrostatic force for $x$-axis does not change by the $x$-displacement and no electrical spring effect occurs. The peak amplitude increased with the dc bias. The electrical responses were mainly dominated by parasitic capacitance and it is difficult to see the response from $x$-axis resonant vibration. The parasitic capacitance is from capacitance between substrate and electrode pads.

(a)

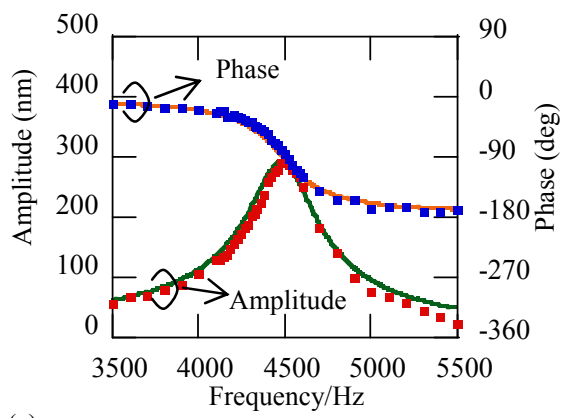

(c)

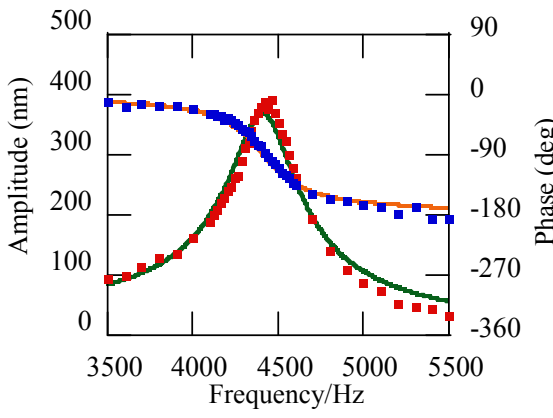

(e)

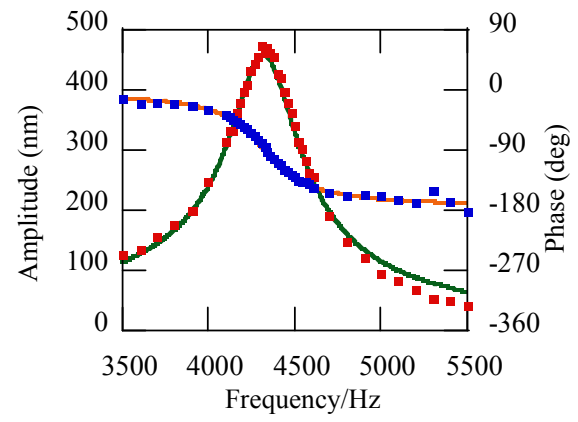

- Measurement __Analysis (b)

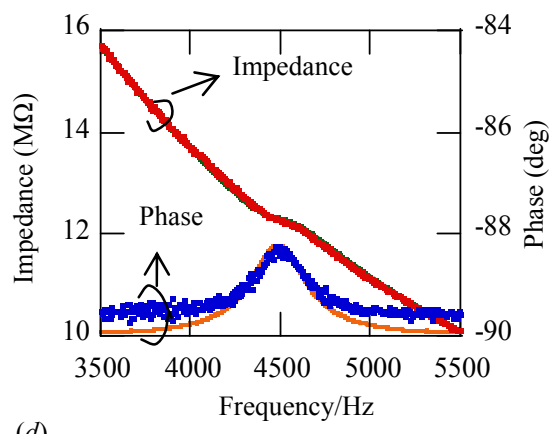

(d)

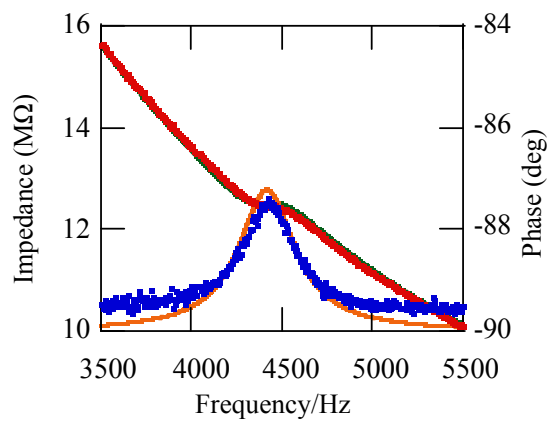

(f)

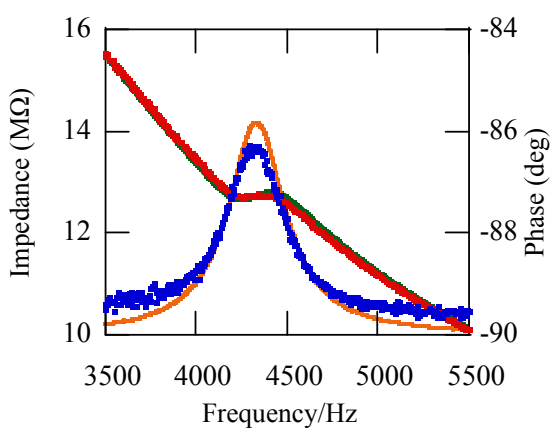

Fig. 7. Mechanical (a, c, e) and electrical (b, d, f) frequency responses around y-axis resonance mode at bias voltage $(\mathrm{a}, \mathrm{b}) 5 \mathrm{~V},(\mathrm{c}, \mathrm{d}) 6 \mathrm{~V},(\mathrm{e}, \mathrm{f}) 7 \mathrm{~V}$ 
The $y$-axis resonant frequency decreased as the dc bias increased and the peak amplitude increased. In figs. 7, the mechanical and electrical frequency responses around $y$-axis resonant frequency at different dc bias voltages are plotted. The resonant frequency shift is caused by the electrostatic spring effect that is the non-linearity of force-displacement relationship. The simulation responses that are obtained with the parameters fitted with measured responses at $E_{\text {bias }}$ of $5 \mathrm{~V}$ agreed well with the measurements even at the different bias voltages as shown in Figs. 7c, 7d, 7e and 7f. As the dc bias increased, the peak amplitude increases and the peak frequency decreases, which describe well the electrical soft spring effect of parallel plate capacitance.

\section{Two-Degree-of-Freedom Decoupled Resonator}

\subsection{Equivalent Circuit Model}

The comb transducer model was applied to a decoupled 2DOF in-plane resonator for analyzing frequency tuning capability and transient responses of comb resonator. In the latter analysis, dc operating point might be changed during analysis, which means the dc bias on comb transducer is not constant. Therefore it is difficult to analyze using conventional model, but the proposed model is applicable.

Single crystal silicon resonators were fabricated on a silicon-on-glass (SOG) structure. In this device, since substrate is insulating material, the parasitic capacitance that dominated electrical impedance characteristics of the SOI resonator in the previous chapter can reduce significantly. The resonators were designed as a simple vibrating gyroscope. The outer and inner frames are oscillated along $x$-axis using comb transducers (fix 1 and fix 2 ). Since the outer frame is movable for only $x$-axis and inner mass is movable for both $x$ and $y$-axes, the inner frame is oscillated along $y$-axis caused by Coriolis force. The Coriolis vibration is detected by parallel plate capacitors (fix 3 and fix4).

The comb transducers (fix 1 and fix2) have a gap of $3 \mu \mathrm{m}$, structure height of $15 \mu \mathrm{m}$, and initial overlap of comb fingers of $20 \mu \mathrm{m}$ and their capacitance are $124 \mathrm{fF}$. Fig. 9 shows the equivalent circuit of the driving structure of the resonator. Table 2 shows the lumped parameter of the resonator at the static pressure of $80 \mathrm{~Pa}$ which were calculated from the design, but they were adjusted to fit the measured resonance frequency (3.1 $\mathrm{kHz}$ ) and quality factor (797).

Table 2. Lumped Parameters of Resonator

\begin{tabular}{ccc}
\hline Mass (drive-system) & $m_{x}$ & $1.15 \times 10^{-8} \mathrm{~kg}$ \\
\hline Spring constant (drive-system) & $k_{x}$ & $1.776 \mathrm{~N} / \mathrm{m}$ \\
\hline Damping (drive-system) & $c_{x}$ & $5.0 \times 10^{-7} \mathrm{Ns} / \mathrm{m}$ \\
\hline Mass (sense-system) & $m_{y}$ & $5.549 \times 10^{-9} \mathrm{~kg}$ \\
\hline Spring constant (sense-system) & $k_{y}$ & $9.38 \mathrm{~N} / \mathrm{m}$ \\
\hline Damping (sense-system) & $c_{y}$ & $1.4 \times 10^{-6} \mathrm{Ns} / \mathrm{m}$ \\
\hline
\end{tabular}



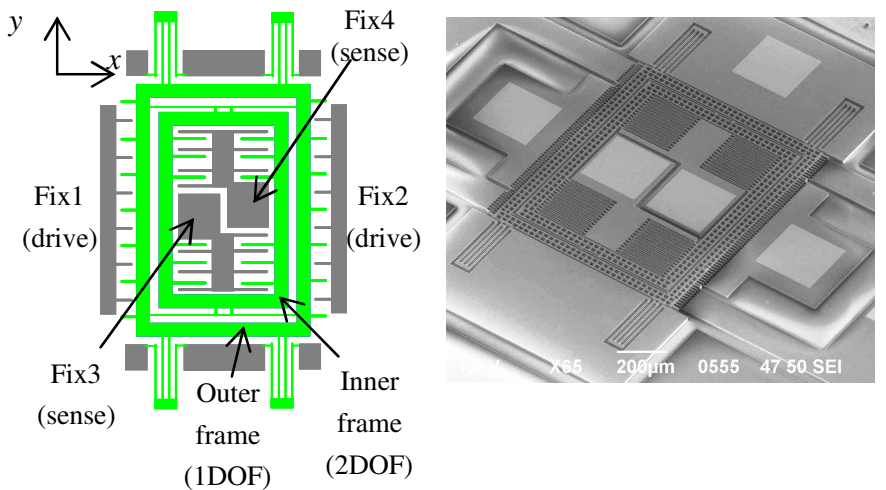

Fig. 8. In-plane decoupled $2 \mathrm{DOF}$ resonator (a) schematics and (b) fabricated device for SOG gyroscope

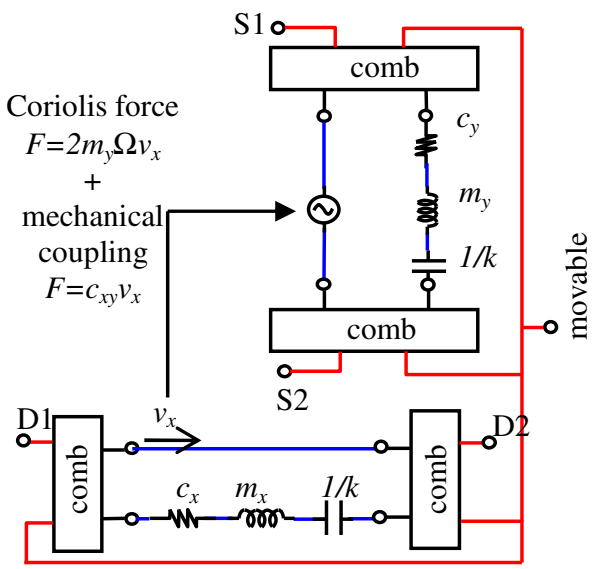

Fig. 9. Equivalent circuit model for decoupled resonator as a gyroscope. Four set of the comb models described in fig. 4 were being used.

\subsection{Frequency Tuning Analysis}

The frequency tuning of two ( $x$ and $y)$ resonant modes are important for gyroscopes. Because of the fabrication error, it is difficult to tune only by structure designing. Therefore, electrical tuning which is described in the previous section is often used. In the present device, the parallel plate capacitors are employed at the comb attached to inner resonator. The capacitors for detecting y-axis displacement can be used for frequency tuning.

The resonance frequencies of y-axis vibration at different voltages were obtained and measured. In the simulation, the electrical port corresponding to the movable electrode was biased, and ac analysis was performed. By probing the current flows on 
the mechanical circuits, the resonant frequencies were evaluated. The $\mathrm{x}$-axis resonant frequency is $1970 \mathrm{~Hz}$ and constant against $E_{\text {bias. }}$. Fig. 10 shows the y-axis resonant frequency in the bias voltage range from 9.5 to $11 \mathrm{~V}$. The simulated and measured resonant frequency agreed well. At bias voltage of $11 \mathrm{~V}$ the measured frequency is slightly lower than Simulation, which may be caused by the non-linear response of the clamped-clamped beams of y-axis resonators.

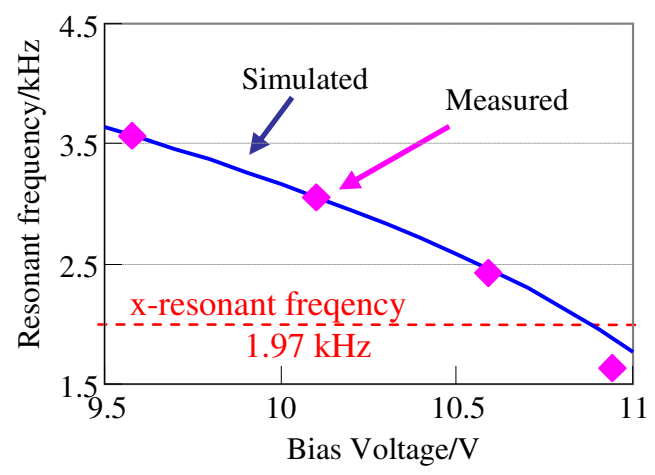

Fig. 10. Simulated and measured resonant frequency shift by the bias voltage

\subsection{Self-induced Oscillation}

In this section, power on response of comb resonator is simulated and measured [5]. We dealt with the outer frame resonator of decoupled resonator device as a simple $x$ axis resonator. The equivalent circuit for $x$-axis was simply modeled as lumped singleaxis resonator with two combs. To validate our model, all the circuit components including op-amps and special function ICs are modeled in the equivalent circuit and the measurement results and the simulation results were compared. The spice models of ICs are provided from manufacturers.

The block diagram of self-induced oscillation circuit is shown in Fig. 11 [6]. The resonator has two comb transducers fix 1 and fix 2 for driving and sensing, respectively. The sensing comb was dc-biased at $E_{\text {bias. }}$. The capacitance change by the $x$-axis displacement generated the current, which was converted to output voltage $V_{\text {out }}$ using a transresistance amplifier (National Semiconductor, LF353). The output voltage is proportional to the vibration velocity. The rms voltage $V_{\mathrm{a}}$ is obtained through demodulator (Analog Devices, AD630) and low pass filter (LPF) with cut off frequency of $20 \mathrm{~Hz}$. In order to attain constant vibration, a variable gain amplifier (VGA; Analog Devices, AD603) controls the loop gain by referring the difference between $V_{\mathrm{a}}$ and dc voltage $V_{\text {set }}$. The gain control range of the VGA is from $-10 \mathrm{~dB}$ to $30 \mathrm{~dB}$. Finally, the output of VGA, $V_{\text {in }}$, is fed back to the driving comb (fix1). 


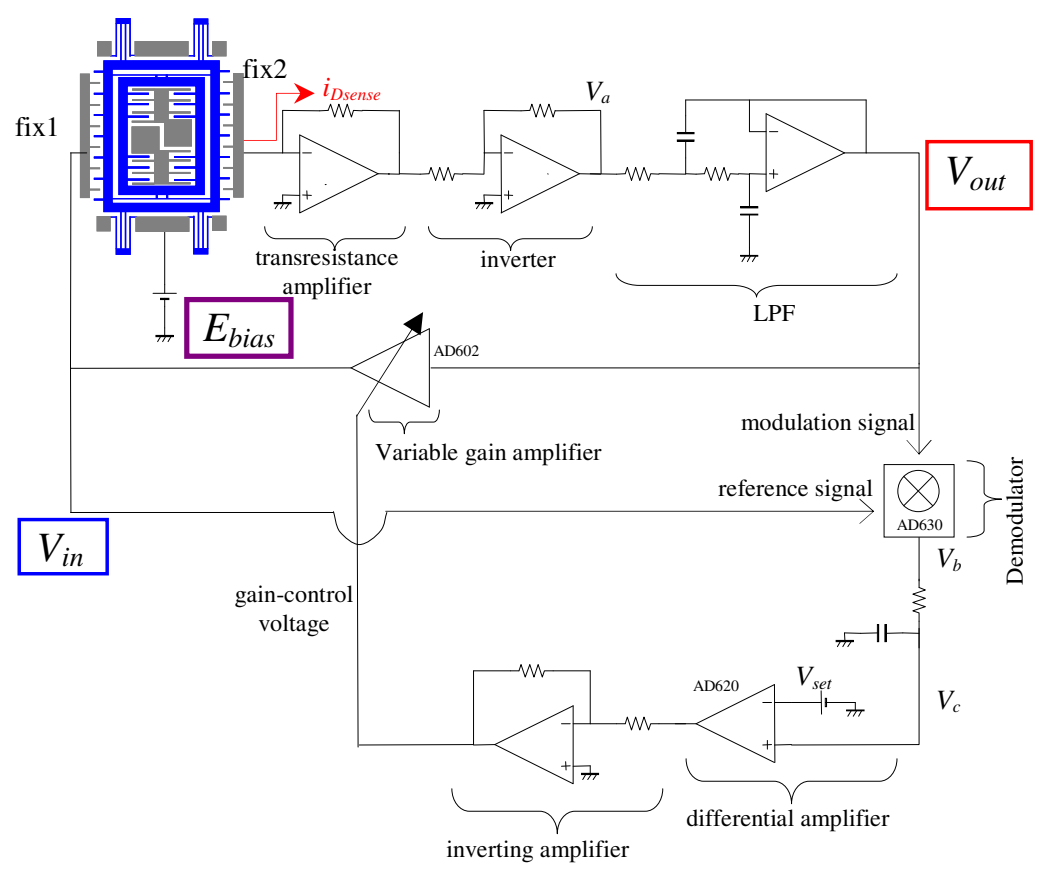

Fig. 11. Schematic diagram of the constant amplitude and self-induced oscillating circuit with electrostatic comb resonator

\subsection{Power on Transient Response Analysis}

Transient response of the self-induced oscillation system was measured and analyzed by changing dc bias voltage $E_{\text {bias }}$ under $V_{\text {set }}=173 \mathrm{mV}$ at the ambient pressure of $80 \mathrm{~Pa}$. $V_{\text {in }}$ and $V_{\text {out }}$ from power-on were monitored both in simulation and measurement. In simulation, transient analysis was done using the circuit simulator. Fig. 12 shows $V_{\text {in }}$ and $V_{\text {out }}$ from power-on (simulation start) at $E_{\text {bias }} 4.78 \mathrm{~V}$. The time from power-on to reaching $V_{\text {in }}$ to $80 \%$ of the saturated amplitude (defined as a risetime). The risetime and the amplitude of the $V_{\text {in }}$ and $V_{\text {out }}$ in stable-state as a function of $E_{\text {bias }}$ are summarized in Fig. 13. The critical bias voltage $E_{\text {bias }}$ for self-induced oscillation in the measurement and analysis were $3.5 \mathrm{~V}$ and $4 \mathrm{~V}$, respectively. The equivalent circuit analysis shows good agreement with the measured response. In addition, we confirm that the amplitude of vibration velocity can be controlled by changing $V_{\text {set }}$ both in the measurement and analysis. 

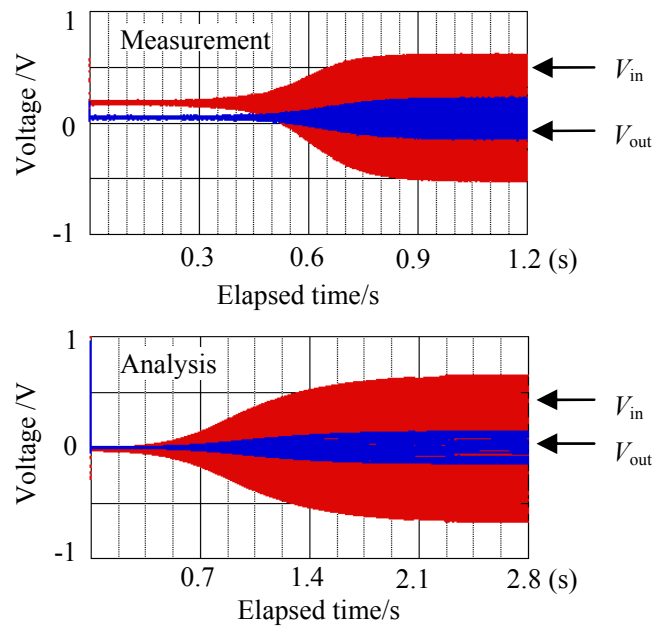

Fig. 12. Transient response of self-induced oscillation under bias $4.78 \mathrm{~V}$
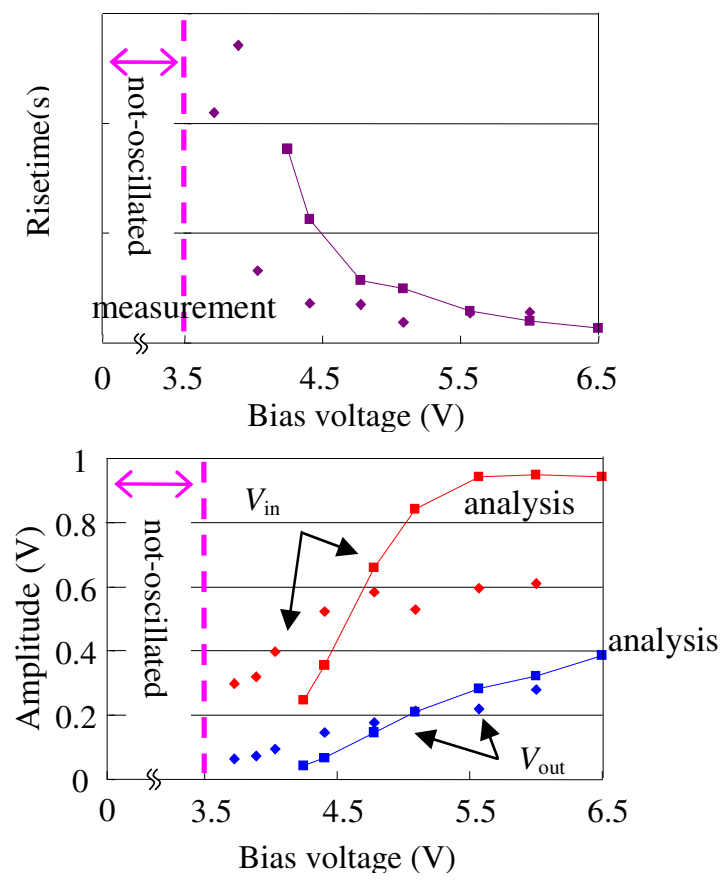

Fig. 13. Power on response time and saturated voltage of both input and output as a function of bias voltage 


\subsection{Discussion}

The lower limit of $E_{\text {bias }}$ for self-induced oscillation was 3.5-4 V. The closed loop gain (total gain) of the circuit is a sum of $G_{1}, G_{2}$ and $G_{3}$. $G_{1}$ is the gain of the MEMS resonator depending on $E_{\text {bias }}$, driving frequency $f$, and damping factor $r . G_{2}$ is the transimpedance amplifier gain, $160 \mathrm{~dB} . \mathrm{G}_{3}$ is the gain of VGA that is changed from -10 to $16 \mathrm{~dB}$. The range of $G_{3}$ is limited by $V_{\text {set }}=173 \mathrm{mV}$ and positive value of $V_{\mathrm{a}}$. The total gain should be larger than $0 \mathrm{~dB}$ at start-up to induce oscillation. After the oscillation starts, $G_{3}$ decreases until the rms amplitude $V_{\text {a }}$ approached to $V_{\text {set }}$. The closed-loop gain becomes $0 \mathrm{~dB}$ when the resonator reaches at its stable-state. The electrostatic force $F$ at the drive comb and the sensitivity of the sensing comb is proportional to $E_{\text {bias }}$. If the $G_{1}$ is lower than $-176 \mathrm{~dB}$ at a low bias voltage, the resonator do not oscillate, since the maximum of $G_{2}+G_{3}$ is $176 \mathrm{~dB}$ and the total gain at start-up is lower than $0 \mathrm{~dB}$. Calculated $G_{1}$ at $E_{\text {bias }} 4 \mathrm{~V}$ is $-173 \mathrm{~dB}$ using the parameters in Table 2, which agreed between the simulated and measured results.

Fig. 14 shows the total gain $G_{1}+G_{2}$ of electrostatic resonator and transimpedance amplifier. The minimum gain at which the oscillation induced is $-15.2 \mathrm{~dB}$ in the simulation, which agreed with the VGA controllable range. In the experiments, the minimum gain is slightly higher at $-13 \mathrm{~dB}$, which may be caused by the noise from the resonator.

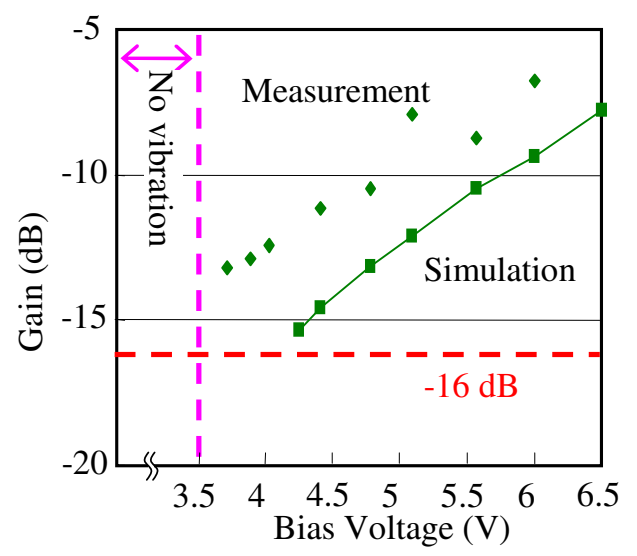

Fig. 14. Simulated and measured total gain of the electrostatic resonator and transimpedance amplifier as a function of bias voltage

In Fig. 12, $V_{\text {in }}$ and $V_{\text {out }}$ differed between simulation and measurement. Also in Fig. 13 , the voltage at which oscillation induced was lower than the simulation, which may be caused by the offset voltages of the op-amps in the circuit. The stray capacitance in the resonator and the change in operating pressure are considered as a main reason. We assumed that the circuit has ideal and no phase shift on $V_{\text {out }}$ against $V_{\text {in }}$ at the resonance frequency. However the stray capacitance caused phase shift in the feedback loop, which resulted in driving frequency shift from the resonance. The driving frequency shift may cause a change in the device gain $G_{1}$ significantly since the resonator driven 
in vacuum had high $Q$-factor. The operating pressure also changes $G_{1}$. Because of the change in $\mathrm{G}_{1}$, the equilibrium position of total gain $=0 \mathrm{~dB}$ was changed and the measured values $V_{\text {in }}$ and $V_{\text {out }}$ were changed to reach total gain at unity.

\section{Conclusion}

In this article, the self-dependent electrical equivalent circuit model of in-plane 2DOF comb transducers was proposed. The model has the dummy spring for detecting the displacement of comb, which allows us to simulate comb drive resonator without modifying the model parameter whenever the device or circuit parameter are modified. The validity of the model is proved through the simulation and measurement of both frequency response of $2 \mathrm{DOF}$ SOI resonator and the frequency tuning and transient response of decoupled SOG resonator. In the frequency response analysis, the effect of bias voltage on the amplitude and frequency on resonance was shown in simulation, which agreed well with the measurements. For the SOG resonator, the frequency tuning by electrostatic spring effect has successfully analyzed. In the transient analysis, the power on response was well analyzed and agreed well with the measurements. The oscillation criteria against the bias voltage were discussed using the simulation results.

Acknowledgments. A part of this work was supported by the Ministry of Education, Science, Sports and Culture, Grant-in-Aid for Young Researchers, 19676002.

\section{References}

1. Nguyen, C.T.-C., Howe, R.T.: An Integrated CMOS Micromechancial resonator High-Q oscillator. IEEE Journal of Solid-State Circuits 34(4), 440-455 (1999)

2. Ayano, K., Suzuki, K., Hashiguchi, G., Fujita, H.: Electromechanical Analysis of a Micromahicned Comb-Drive Actuator by Admittance Measurement. IEE J. Trans. SM 126(7), 282-285 (2006)

3. Oiso, H., Kariya, S., Hashiguchi, G.: A proposal of gyroscope sensor using asymmetrical comb drive actuator. In: SICE Annual Conference 2007, September 17-20, pp. 2935-2939. Kagawa University, Japan (2007)

4. Tokusaki, H., Hirai, Y., Sugano, K., Tsuchiya, T., Tabata, O.: Component modeling of 2DOF comb transducer for equivalent circuit using built-in displacement detection. In: APCOT 2010, Perth, Australia, July 6-9, p. 160 (2010)

5. Tokusaki, H., Hirai, Y., Sugano, K., Tsuchiya, T., Tabata, O.: Equivalent circuit analysis of micromechanical resonator using comb transducer model with built-in displacement detection. In: IEEE NEMS 2011, Kaohsiung, Taiwan, February 20-23, pp. 1288-1292 (2011)

6. Alper, S.E., Temiz, Y., Akin, T.: A Compact Angular Rate Sensor System Using a Fully Decoupled Silicon-on-Glass MEMS Gyroscope. Journal of Microelectromechanical Systems 17(6), 1418-1429 (2008) 